\title{
Psychological distress and postponed fertility care during the COVID-19 pandemic
}

\author{
Angela K. Lawson $^{1}$ (D) Dana B. McQueen ${ }^{1}$ (D) Amelia C. Swanson ${ }^{1}$ (D) $\cdot$ Rafael Confino $^{1}$ (D) $\cdot$ Eve C. Feinberg ${ }^{1} \cdot$ \\ Mary Ellen Pavone ${ }^{1,2}$ (B)
}

Received: 8 October 2020 / Accepted: 24 November 2020 / Published online: 5 January 2021

(C) Springer Science+Business Media, LLC, part of Springer Nature 2021

\begin{abstract}
Purpose To evaluate perceptions of delayed fertility care secondary to the COVID-19 pandemic.

Methods This was a cross-sectional anonymous survey of $N=787 / 2,287$ patients (response rate $=42.6 \%$ ) from a single academic fertility center. Participants were randomized 1:1 to receive supplemental educational explaining the rationale behind recommendations to delay fertility treatments due to the COVID-19 pandemic. Assessment of well-being was conducted via the Personal Health Questionnaire Depression Scale, the Generalized Anxiety Disorder-7, the Ways of Coping-Revised, the Appraisal of Life Events Scale, and influence of supplemental education on agreement with ASRM COVID-19 Taskforce recommendations and associated distress.

Results Participants in the education v. no education groups were $35.51(\mathrm{SD}=4.06)$ and $37.24(\mathrm{SD}=5.34)$ years old, married $(90.8 \%$ v. $89.8 \%)$, had a graduate degree $(53.9 \%$ v. $55.4 \%),>1$ year of infertility $(73.4 \%$ v. $74.4 \%)$, and were nulliparous $(69.0 \%$ v. $72.6 \%)$, with moderate to high distress $(64.9 \%$ v. $64.2 \%)$ (ns). Distress was related to age, duration of infertility, and engagement in social support seeking and avoidant coping strategies $(P<0.001)$. Agreement with recommendations was related to receipt of supplemental education, history of pregnancy loss, and use of cognitive coping $(P=0.001)$.

Conclusion Most participants were distressed by the delay of treatments. Supplemental education increased acceptance of recommendations but did not decrease distress. Future treatment delays should include education related to and assessment of understanding of recommendations, and inclusion of mental health professionals in patient care.
\end{abstract}

Keywords Coronavirus $\cdot$ COVID $\cdot$ Fertility $\cdot$ In vitro fertilization $\cdot$ Distress

\section{Introduction}

The coronavirus disease 2019 (COVID-19) spread across the globe and caused worldwide disruptions in daily living beginning in late 2019 through 2020 and has no discernable ending date. The pandemic has led to legally mandated physical distancing, mass closures of businesses and public spaces, increased use of personal protective equipment both within and outside healthcare facilities, and large increases in unemployment (from $3.6 \%$ in January 2020 to $>14 \%$ in April 2020) [1]. At the time of the current study (May 2020), there

Mary Ellen Pavone

MaryEllen.Pavone@nm.org

1 Department of Obstetrics and Gynecology, Northwestern University Feinberg School of Medicine, Chicago, IL 60611, USA

2 Chicago, USA were a million confirmed cases of COVID-19 and sixty thousand deaths in the USA. These numbers likely reflect an underestimate of the total numbers of cases and deaths due to issues related to lack of testing and problems with reporting [2]. In this unprecedented public health crisis, evidence of increased emotional distress in healthcare providers and the general population has also been abundant. Fears related to risk of contracting COVID-19 and physical distancing orders have led to social isolation and financial strain that was associated with feelings of anxiety and depression [3, 4].

In March and April of 2020, efforts to ensure an adequate supply of ventilators and conserve healthcare resources for medical professionals caring for patients with COVID-19 resulted in recommendations to limit non-emergency medical care and surgical procedures by 49 medical professional societies in the USA as well as state and local governments. Similar recommendations were made across the globe by every major medical society. 
Although infertility is a disease and fertility treatments are often necessary to achieve pregnancy, such treatments require multiple in-person interactions involving multi-disciplinary teams of healthcare professionals and staff, and the proximity required for ultrasounds, blood work, and procedures prohibits effective physical distancing. Infertility is also highly prevalent and the number of patients seeking treatment at any given time poses an increased risk of exposure for both patients and healthcare providers working in fertility clinics.

Due to global concerns related to conservation of resources and risk exposure to patients and clinic personnel, on March 17, 2020, the COVID-19 Taskforce of the American Society for Reproductive Medicine (ASRM) issued a recommendation to suspend initiation of all new treatment cycles, including ovulation induction, intrauterine inseminations (IUI), and in vitro fertilization (IVF) including retrievals and frozen embryo transfers. In addition, they recommended strongly considering cancellation of all embryo transfers, while continuing to care for patients who were currently "incycle" or who required emergency stimulation and cryopreservation for fertility preservation prior to gonadotoxic chemotherapy or extirpative surgery [5]. This statement was comparable to a statement put forth by the European Society for Human Reproduction and Embryology (ESHRE) as well as multiple other similar societies. As a result of these recommendations, and in accordance with state and local governance, most fertility clinics nationwide stopped all nonemergency fertility treatment cycles and elective surgery.

Although the COVID-19 pandemic was rapidly spreading, the death toll was rapidly escalating and news reports of overburdened medical facilities were abundant; some physicians and patients reported that they were shocked or distressed by the recommendation to temporarily pause treatment $[6$, 7]. Given the limited amount of time between the release of the national and international recommendations related to delaying fertility care and contact with patients regarding the cancellation or delay of their treatment, it is unclear if patients fully understood and/or accepted the rationale for delaying fertility care. Acceptance of the rationale for delaying fertility care may be complicated not only by a lack of sufficient information related to the rationale for delaying care but also by patient's prior experiences of infertility.

Individuals pursuing fertility treatment have often been trying to conceive a child for months to years prior to seeking care in a fertility clinic [8]. The often lengthy duration of infertility and its treatment has repeatedly been associated with increased risk of psychological distress [9]. Further, psychological distress has been shown to affect medical decision making $[10,11]$. It is therefore likely that any additional delay to fertility care, such as the delay, which followed the ASRM COVID-19 Taskforce and international societies recommendations as well as state mandates, could increase the risk of psychological distress, depression, and anxiety and influence patient's decision-making strategies. Indeed, a recent survey of 518 fertility patients found that $85 \%$ of study participants were moderately to extremely distressed by their fertility cycle cancellation, regardless of treatment type, due to the COVID19 pandemic. Nearly a quarter of participants found the delay to be as distressing as the loss of a child. Only approximately a third of patients agreed with the recommendation to cancel all fertility treatment cycles and $52 \%$ would have continued their treatment despite the risks of the pandemic [12].

The aim of this study was to assess perceptions related to the recommended pause in fertility care and the emotional well-being among fertility patients. A secondary aim was to assess if supplemental education, demographics, or social variables influenced patient perceptions.

\section{Materials and methods}

\section{Study design}

This was a single-center cross-sectional study performed in the Division of Reproductive Endocrinology and Infertility at Northwestern University Feinberg School of Medicine. All patients whose email address was included in the electronic medical record and were seen for treatment or follow-up between February 01, 2020, and April 21, 2020 were electronically invited to participate in an anonymous survey via Survey Monkey. There were no exclusion criteria for study enrollment. Forty-nine patients did not have an identified email address; 2287 patients were emailed, 1848 opened their survey invitation, and 787 (42.6\% response rate) completed all $(n=599)$ or part $(n=188)$ of the survey. A follow-up message was sent to patients 2 days after the first message. In order to capture patients during the same discrete period of time, the survey was sent on April 29, 2020 and closed on May 07, 2020. No identifying information was obtained on participants. Institutional Review Board (IRB) approval was obtained.

\section{Education intervention}

Participants were randomized 1:1 to receive additional education regarding the rationale behind the ASRM COVID-19 Taskforce recommendations to delay fertility treatment during the COVID-19 pandemic. This 1-page graphic design image included 4 images with 2 sentences per image describing (1) "Why Are Treatments Delayed" [ because of attempts to conserve resources including personal protective equipment, anesthesiologists, and nurses], (2) "How Does My Treatment Affect Public Health" [ a high number of personal interactions are necessary during fertility treatments and these interactions prohibit effective physical distancing and increase risk of infections], (3) "Are Other Patients Affected" [ other types of patients may have experienced delayed care secondary to the 
COVID-19 pandemic, including those requiring joint replacement, gynecologic or transplant surgery], and (4) "When Will Treatments Resume and Will This Impact My Chances of Having a Baby?" [i.e., participants were reassured that up to a three-month delay in care should not impact their long-term chances of success with fertility treatment].

\section{Measures}

In addition to demographic variables, history of engagement in fertility treatment, and items assessing perceptions related to fertility treatment delay, study participant's emotional wellbeing was assessed through several empirically validated measures. Participants completed questions on demographics and psychological measures first. Once these were completed, the survey went on to assess perceptions related to the ASRM COVID-19 Taskforce recommendations to pause fertility treatment, methods of coping, cognitive appraisals of the cancellations, and associated cancellation distress (measured with a single-item question). Individuals randomized to receive the education intervention viewed this information before any items related to the cancellation or delay of fertility treatments. Individuals randomized to no supplemental education proceeded to items related to cancellation or delay of fertility treatments.

\section{Appraisal of Life Events scale}

The Appraisal of Life Events (ALE) [13] scale assesses three dimensions of cognitive appraisal (i.e., perceptions) of specific life events. Two of the dimensions (i.e. Threat and Loss) were used to assess participant appraisal of cycle cancellation/ delay. The third dimension (i.e., Challenge) was excluded as it assesses positive reactions and such reactions were not expected from the cancellation/delay of treatment. Higher scores on the ALE indicate greater dimensional appraisal.

\section{Generalized Anxiety Disorder-7 scale}

The Generalized Anxiety Disorder-7 (GAD-7) scale [14] includes 7 items which assess for symptoms of anxiety in the last 2 weeks. A higher scale score indicates greater anxiety with scores of $0-4$ associated with minimal anxiety, 5-9 mild anxiety, 10-14 moderate anxiety, and scores of 15-21 associated with severe anxiety.

\section{Patient Health Questionnaire-8}

The Patient Health Questionnaire-8 (PHQ-8) [15] is an 8-item measure which assesses the presence of symptoms of major depressive disorder and contains all but a single item assessing suicidal ideation from the PHQ-9 [16]. Higher scores on the scale indicate greater symptoms of depression and scores $\geq 10$ are suggestive of clinically significant depression.

\section{Ways of Coping-Revised scale}

Twenty-seven items of the Ways of Coping-Revised (WOC$\mathrm{R})$ scale [17] were used to assess three types of coping: selfblame and avoidance [SBA], informational and emotional support seeking [IES], and cognitive restructuring [CR]). Higher scores on the WOC-R subscales reflect greater use of the coping strategy.

\section{Data analysis}

Statistical analyses were performed with the use of SPSS (Version 26.0) (IBM) using parametric tests for normally distributed data and nonparametric tests for non-normally distributed data, unequal sample variances, categorical data, and/or comparisons with small sample sizes. Ordinal logistic regression analyses were used to test the models predicting agreement with recommendations to delay treatment and resulting distress levels. Analyses were based on available data, sample sizes are provided, and $P<0.05$ ( 2 tailed) was considered to be significant.

\section{Results}

\section{Demographics}

Of the 787 number of respondents, 648 were female, 48 were male, and sex was not available for 91 participants. Due to the small number of male respondents, these surveys were excluded from analysis. Of the total sample who were sent a survey, $n=1134$ participants self-identified as White, $n=158$ as Asian, $n=140$ as Black or African American, $n=7$ as American Indian or Alaska Native, $n=8$ as Native Hawaiian or Other Pacific Islander, $n=275$ as some other racial ethnic background, and $n=390$ elected not to answer. There were no statistically significant demographic differences between the two groups with respect to age [educational intervention $35.51(\mathrm{SD}=4.06)$ vs no intervention $37.24(\mathrm{SD}=$ 5.34)] or other demographic variables. The majority of women in both groups were married or partnered with a male, had no children, a graduate level degree, health insurance, and a household income greater than $\$ 100,000$ per year. Most participants had been trying to conceive for greater than a year, had been under the care of an Reproductive Endocrinology and Infertility physician for greater than 6 months, and reported believing that their chances of having a baby with fertility treatment was $\geq 50 \%$. Slightly less than a half of women reported a prior pregnancy and of those women, greater than $40 \%$ reported a prior pregnancy loss (Table 1 ). 
Table 1 Demographic characteristics of female participants who received $(n=271)$ or did not receive $(n=285)$ the supplemental education

\begin{tabular}{lll}
\hline Variable & Intervention & No intervention \\
& $N(\%)$ & $N(\%)$ \\
\hline
\end{tabular}

Age (mean, SD)
Participant age
Partner's age
Partner's sex
Female
Male
Unknown
Marital status
Single (never married)
Married/partnered
Widowed
Divorced
Unknown

Desired Number of children ${ }^{\mathrm{a}}$

0
1
2
3
$4+$
Unknown
TTC
$0-6$ months
6 months-1 year
$1-2$ years
$2+$ years
Unknown

\section{Fertility care duration}

0-6 months

6 months -1 year

$1-2$ years

$2+$ years

Unknown

Prior pregnancy

No

Yes

Pregnancy loss

No (gravidity $\geq 1$ )

No (gravidity $=0$ )/unknown

Yes

Number of children

0 (never pregnant)

1

2

$3+$

Unknown

Prior birth with fertility tx

$\begin{array}{lll}\text { No } & 81(29.9) & 87(30.5) \\ \text { Yes } & 82(30.3) & 78(27.4) \\ \text { No prior births/unknown } & 108(39.9) & 120(42.1)\end{array}$

Table 1 (continued)

\begin{tabular}{|c|c|c|}
\hline Variable & $\begin{array}{l}\text { Intervention } \\
N(\%)\end{array}$ & $\begin{array}{l}\text { No intervention } \\
N(\%)\end{array}$ \\
\hline 0 & $158(58.3)$ & $182(63.9)$ \\
\hline$\geq 1$ & $66(24.4)$ & $55(19.3)$ \\
\hline Ūnknown & $47(17.3)$ & $48(16.8)$ \\
\hline \multicolumn{3}{|l|}{ Education } \\
\hline High school degree & $7(2.6)$ & $9(3.2)$ \\
\hline Associate's degree & $9(3.3)$ & $13(4.6)$ \\
\hline Bachelor's degree & $109(40.2)$ & $105(36.8)$ \\
\hline Master's degree & $100(36.9)$ & $108(37.9)$ \\
\hline Doctorate/professional degree & $46(17.0)$ & $50(17.5)$ \\
\hline \multicolumn{3}{|l|}{ Household income } \\
\hline$<\$ 50,000$ & $4(1.5)$ & $7(2.5)$ \\
\hline$\$ 50,000-\$ 100,000$ & $46(17.0)$ & $42(14.7)$ \\
\hline$\$ 100,000-\$ 200,000$ & $109(40.2)$ & $105(36.8)$ \\
\hline$>\$ 200,000$ & $109(40.2)$ & $129(45.3)$ \\
\hline Unknown & $3(1.1)$ & $2(0.7)$ \\
\hline \multicolumn{3}{|l|}{ Health insurance } \\
\hline No & $0(0.0)$ & $1(0.4)$ \\
\hline Yes & $271(100.0)$ & $284(99.6)$ \\
\hline \multicolumn{3}{|l|}{ Insurance covered evaluation } \\
\hline Full coverage & $146(53.9)$ & $152(53.3)$ \\
\hline No coverage & $16(5.9)$ & $17(6.0)$ \\
\hline Partial coverage & $86(31.7)$ & $84(29.5)$ \\
\hline Unknown & $21(7.7)$ & $32(11.2)$ \\
\hline \multicolumn{3}{|l|}{ Insurance covered treatment } \\
\hline Full coverage & $99(36.5)$ & $110(38.6)$ \\
\hline No coverage & $30(11.1)$ & $36(12.6)$ \\
\hline Partial coverage & $132(48.7)$ & $119(41.8)$ \\
\hline Unknown & $10(3.7)$ & $20(7.0)$ \\
\hline \multicolumn{3}{|l|}{ Chance of success beliefs } \\
\hline$<5 \%$ & $17(6.3)$ & $9(3.2)$ \\
\hline$<15 \%$ & $21(7.7)$ & $23(8.1)$ \\
\hline$<25 \%$ & $35(12.9)$ & $34(11.9)$ \\
\hline$<50 \%$ & $43(15.9)$ & $60(21.1)$ \\
\hline $50 \%-75 \%$ & $77(28.4)$ & $90(31.6)$ \\
\hline $75 \%+$ & $77(28.4)$ & $65(22.8)$ \\
\hline Unknown & $1(0.4)$ & $4(1.4)$ \\
\hline
\end{tabular}

Numbers may not total to $100 \%$ due to rounding. There were no significant group differences in demographics. TTC time trying to conceive, $T x$ treatment

${ }^{a}$ Study participants include all clinic patients regardless of whether or not they were seeking fertility treatment

\section{Psychological assessments}

Results from the GAD-7 and PHQ-8 indicate that all participants reported experiencing symptoms of anxiety and depression. Greater than half of participants reported experiencing moderate to severe symptoms of anxiety $(69.1 \%$ intervention group, $73.7 \%$ no intervention group) and most participants 
reported clinically significant symptoms of depression (77.5\% intervention group, $77.9 \%$ no intervention group). There were no group differences in anxiety, depression, appraisal, or coping scores (Table 2).

\section{Agreement with recommendations to pause treatment}

More than half of all participants agreed with or were neutral about the recommendations to delay treatment. Agreement with the cancellation of all fertility treatment cycles, IUI cycles, IVF with the intention to undergo fresh transfer or freeze all, frozen embryo transfer, and egg freezing significantly differed between the intervention and no intervention groups with greater agreement in the intervention group. There were no differences between groups with agreement in cancellation of their own cycle, desire to continue treatment despite the risks associated with the COVID-19 pandemic, the cancellation of cycles for women who were older than 40 or who had a diagnosis of diminished ovarian reserve, and the degree of distress associated with treatment cancellation (as measured by a single-item question) (Table 3 ). The degree of distress associated with cycle cancellation or delay did not differ between intervention groups: no distress (3.3\% intervention group, $1.8 \%$ no intervention group), mild distress (22.1\% intervention group, $11.9 \%$ no intervention group), moderate distress (32.8\% intervention group, $29.8 \%$ no intervention group), and extreme distress (32.1\% intervention group, $34.4 \%$ no intervention group) (Fig. 1).

Table 2 Psychological characteristics of participants who did and did not receive the supplemental education

\begin{tabular}{lll}
\hline Variable & $\begin{array}{l}\text { Intervention } \\
\text { Mean (SD) }\end{array}$ & $\begin{array}{l}\text { No intervention } \\
\text { Mean (SD) }\end{array}$ \\
\hline ALE & $11.37(5.72)$ & $11.29(5.67)$ \\
Loss & $15.98(8.69)$ & $16.50(8.48)$ \\
Threat & $12.90(4.82)$ & $13.11(4.91)$ \\
GAD-7 & $0(0.0)$ & $0(0.0)$ \\
$0-4$ (minimal) & $83(30.7)$ & $73(26.3)$ \\
$5-9$ (mild) & $107(39.6)$ & $121(43.5)$ \\
$10-14$ (moderate) & $80(29.5)$ & $84(30.2)$ \\
$15-21$ (severe) & $13.85(4.83)$ & $13.87(4.74)$ \\
PHQ-8 & $210(77.5)$ & $222(77.9)$ \\
Score $\geq 10$ & & $29.13(8.69)$ \\
WOC-R & $29.28(9.26)$ & $13.54(3.42)$ \\
Self-blame/avoidance & $13.49(3.83)$ & $10.19(2.90)$ \\
Support seeking & $10.36(2.98)$ & \\
Cognitive restructuring &
\end{tabular}

Numbers may not total to $100 \%$ due to rounding. ALE Appraisal of Life Events scale, GAD-7 Generalized Anxiety Disorder-7 scale, $P H Q-8$ Patient Health Questionnaire-8, WOC-R Ways of Coping-Revised scale
A priori hypotheses based on published research with fertility patients were tested to assess relationships between demographic, psychologic, coping variables, and outcome variables of interest (i.e., cancellation distress and agreement with recommendations to cancel/delay fertility treatments). Variables assessing acceptance of recommendations and resulting distress were recoded such that higher scores indicate greater acceptance (e.g., "disagree" was coded as 1, "neutral"/"undecided" as 2, and "agree" as 3).

First, Spearman correlations between the psychologic measures, demographic items, appraisal, coping, and outcome variables were determined (Supplemental Table 4). Only those variables with significant correlation coefficients which preceded the randomized education (e.g., demographic items and psychologic measures) or were not expected to be affected by the educational information (i.e., WOC) provided in the survey were included as independent variables in subsequent regression analyses. The PHQ-8 was not included in models where the GAD7 was included due to high multicollinearity between the variables. Finally, for parsimony, only the model predicting agreement with cancelling all types of fertility treatments was tested due to similarities with the other types of agreement with cycle/treatment cancellation.

We conducted ordinal logistic regression to examine the ability of our measures to predict agreement with recommendations to delay treatment and subsequent distress. Group (i.e., intervention vs. no intervention) was included in the models because we wanted to better understand the role of the educational intervention as well as demographic, psychologic, and coping variables on overall agreement with recommendations to delay treatment and associated distress.

Evaluation of the overall model for level of distress related to the cancellation or delay of fertility treatments which included the educational randomization variable (dummy coded), age, how many months the participant had been trying to conceive (TTC), use of social support coping strategies (WOCIES) or avoidance strategies (WOCSBA), and GAD7 was significant $\left(\chi^{2}[n=471]=115.14 ; \mathrm{df}=6, P<0.001\right)$. The Pearson goodness-of-fit statistic was not significant $(P=$ $0.174)$, suggesting a good model fit. The odds of being more distressed by the cancellation/delay of fertility treatments was related to younger age [odds ratio [OR] $0.959,95 \%$ confidence interval [CI] 0.920-1.00, Wald $\chi^{2}(1)=3.868, P=$ 0.049], longer TTC [odds ratio [OR] 1.32, 95\% confidence interval [CI] 1.10-1.58, Wald $\left.\chi^{2}(1)=9.209, P=0.002\right]$, higher scores on WOCIES [odds ratio [OR] 1.07, 95\% confidence interval [CI] 1.01-1.13, Wald $\left.\chi^{2}(1)=5.53, P=0.019\right]$, and WOCSBA subscales [odds ratio [OR] 1.08, 95\% confidence interval [CI] 1.05-1.11, Wald $\chi^{2}(1)=29.99, P<$ 0.001]. Nagelkerke $R^{2}$, a measure of strength of association between the predictors and the dependent variable, was 0.23 for the entire model. 
Table 3 Effect of COVID-19 pandemic on fertility-related concerns

\begin{tabular}{lll}
\hline Variable & Intervention & No intervention \\
& $N(\%)$ & $N(\%)$ \\
\hline
\end{tabular}

Lost job
No
Yes

Insurance changed

No

Yes, it will get better

Yes, it will get worse

Unknown

Active fertility cycle cancelled

$\begin{array}{lll}\text { No } & 168(62.0) & 183(64.2) \\ \text { Yes } & 102(37.6) & 101(35.4) \\ \text { Unknown } & 1(0.4) & 1(0.4) \\ \text { Type of treatment postponed } & & \\ \text { None } & 38(14.0) & 54(18.9) \\ \text { TI with oral medications } & 14(5.2) & 11(3.9) \\ \text { IUI with oral medications } & 33(12.2) & 38(13.3) \\ \text { IUI with injectable medication } & 20(7.4) & 22(7.7) \\ \text { IVF egg retrieval with fresh transfer } & 36(13.3) & 23(8.1) \\ \text { IVF egg retrieval with plan to freeze all } & 26(9.6) & 31(10.9) \\ \text { Embryo transfer } & 63(23.2) & 56(19.6) \\ \text { Egg freezing } & 8(3.0) & 6(2.1) \\ \text { Surgery } & 5(1.8) & 6(2.1) \\ \text { Unknown/other } & 28(10.3) & 38(13.3) \\ \text { Belief cancellation affects chances } & & \\ \text { None } & 88(32.5) & 75(26.3) \\ \text { A little bit } & 99(36.5) & 117(41.1) \\ \text { A moderate amount } & 45(16.6) & 51(17.9) \\ \text { A significant amount } & 29(10.7) & 33(11.6) \\ \text { Unknown } & 10(3.7) & 9(3.2)\end{array}$

Agreement with cancelling all fertility cycles ${ }^{\mathrm{a}}$

\begin{tabular}{|c|c|c|}
\hline Agree & $92(33.9)$ & $76(26.7)$ \\
\hline Neutral & $78(28.8)$ & $69(24.2)$ \\
\hline Disagree & $99(36.5)$ & $137(48.1)$ \\
\hline Unknown & $2(0.07)$ & $3(1.1)$ \\
\hline \multicolumn{3}{|c|}{ Medicated timed intercourse } \\
\hline Agree & $57(21.0)$ & $46(16.1)$ \\
\hline Neutral & $87(32.1)$ & $97(34.0)$ \\
\hline Disagree & $126(46.5)$ & $138(48.4)$ \\
\hline Unknown & $1(0.4)$ & $4(1.4)$ \\
\hline \multicolumn{3}{|c|}{ Intrauterine insemination $^{\mathrm{b}}$} \\
\hline Agree & $105(38.7)$ & $84(29.5)$ \\
\hline Neutral & $92(33.9)$ & $81(28.4)$ \\
\hline Disagree & $72(26.6)$ & $114(40.0)$ \\
\hline Unknown & $2(0.7)$ & $6(2.1)$ \\
\hline \multicolumn{3}{|l|}{ IVF-fresh ${ }^{b}$} \\
\hline Agree & $120(44.3)$ & $100(35.1)$ \\
\hline Neutral & $77(28.4)$ & $71(24.9)$ \\
\hline Disagree & $72(26.6)$ & $110(38.6)$ \\
\hline
\end{tabular}

Table 3 (continued)

\begin{tabular}{|c|c|c|}
\hline Variable & $\begin{array}{l}\text { Intervention } \\
N(\%)\end{array}$ & $\begin{array}{l}\text { No intervention } \\
N(\%)\end{array}$ \\
\hline Unknown & $2(0.7)$ & $4(1.4)$ \\
\hline \multicolumn{3}{|c|}{ IVF-freeze all ${ }^{\mathrm{c}}$} \\
\hline Agree & $118(43.5)$ & $95(34.0)$ \\
\hline Neutral & $76(28.0)$ & $62(21.8)$ \\
\hline Disagree & $75(27.7)$ & $122(42.8)$ \\
\hline Unknown & $2(0.7)$ & $4(1.4)$ \\
\hline \multicolumn{3}{|c|}{ Egg freezing ${ }^{\mathrm{b}}$} \\
\hline Agree & $110(40.6)$ & $88(30.9)$ \\
\hline Neutral & $81(29.9)$ & $74(26.0)$ \\
\hline Disagree & $75(27.7)$ & $119(41.8)$ \\
\hline Unknown & $5(1.8)$ & $4(1.4)$ \\
\hline \multicolumn{3}{|c|}{ Frozen embryo transfer ${ }^{\mathrm{b}}$} \\
\hline Agree & $112(41.3)$ & $87(30.5)$ \\
\hline Neutral & $75(27.7)$ & $74(26.0)$ \\
\hline Disagree & $78(28.8)$ & $120(42.1)$ \\
\hline Unknown & $6(2.2)$ & $4(1.4)$ \\
\hline \multicolumn{3}{|l|}{ Surgeries } \\
\hline Agree & $109(40.2)$ & $90(31.6)$ \\
\hline Neutral & $89(32.8)$ & $108(37.9)$ \\
\hline Disagree & $71(26.2)$ & $83(29.1)$ \\
\hline Unknown & $2(0.7)$ & $4(1.4)$ \\
\hline \multicolumn{3}{|c|}{ Diagnostic procedures } \\
\hline Agree & $103(38.0)$ & $89(31.2)$ \\
\hline Neutral & $100(36.9)$ & $109(38.2)$ \\
\hline Disagree & $66(24.4)$ & $84(29.5)$ \\
\hline Unknown & $2(0.7)$ & $3(1.1)$ \\
\hline \multicolumn{3}{|c|}{ Would have preferred option to start } \\
\hline Yes & $157(57.9)$ & $173(60.7)$ \\
\hline No & $37(13.7)$ & 33 (11.6) \\
\hline Uncertain & $50(18.5)$ & $46(16.1)$ \\
\hline Unknown & $27(10.0)$ & $33(11.6)$ \\
\hline \multicolumn{3}{|c|}{ Would continue despite pandemic } \\
\hline Yes & $147(54.2)$ & $155(54.4)$ \\
\hline No & $38(14.0)$ & $37(13.0)$ \\
\hline Uncertain & $61(22.5)$ & $61(21.4)$ \\
\hline Unknown & $25(9.2)$ & $32(11.2)$ \\
\hline \multicolumn{3}{|c|}{ Shouldn't CANCEL DOR or age $40+$} \\
\hline Yes & $156(57.6)$ & $175(61.4)$ \\
\hline No & $14(5.2)$ & $12(4.2)$ \\
\hline Uncertain & $74(27.3)$ & $64(22.5)$ \\
\hline Unknown & $27(10.0)$ & 34 (11.9) \\
\hline
\end{tabular}

Numbers may not total to $100 \%$ due to rounding. DOR diminished ovarian reserve.

${ }^{\mathrm{a}} p<0.05$

${ }^{\mathrm{b}} p<0.01$

${ }^{\mathrm{c}} p<0.001$ 
Fig. 1 Perceived distress as a result of cycle cancellation or delay (excludes missing data)

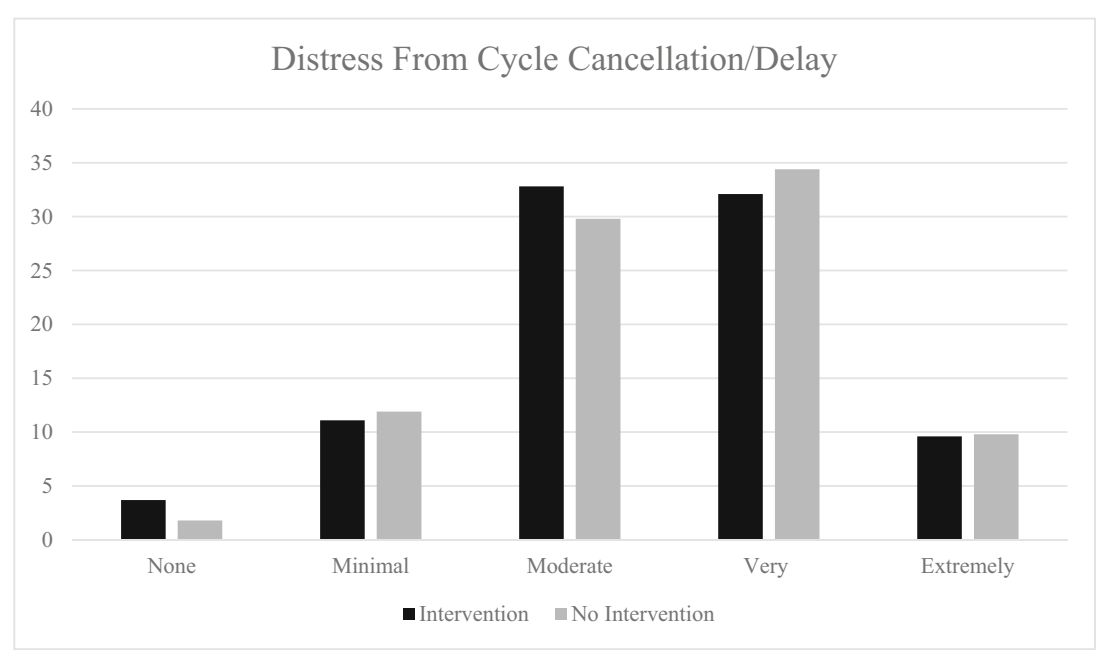

Evaluation of the overall model for agreement with the recommendation to cancel/delay all fertility treatments which included the educational randomization variable (dummy coded), age, history of pregnancy loss, cancellation/delay of their own fertility treatment cycle, use of social support coping strategies (WOCIES), avoidance strategies (WOCSBA), coping via use of cognitive restructuring (WOCCR), and GAD7 was significant $\left(\chi^{2}[n=320]=25.23 ; \mathrm{df}=8, P=0.001\right)$. The Pearson goodness-of-fit statistic was not significant $(P=$ $0.410)$, suggesting a good model fit. The odds of agreeing with the recommendations to cancel/delay all treatment cycles was related to receipt of the educational intervention [odds ratio $[\mathrm{OR}] 1.54,95 \%$ confidence interval [CI] 1.01-2.34, Wald $\left.\chi^{2}(1)=4.06, P=0.044\right]$, a positive history of pregnancy loss [odds ratio [OR] 1.78, 95\% confidence interval [CI] 1.092.90 , Wald $\left.\chi^{2}(1)=5.39, P=0.02\right]$, and higher scores on WOCCR [odds ratio [OR] 1.09, 95\% confidence interval [CI] 1.01-1.18, Wald $\left.\chi^{2}(1)=4.78, P=0.029\right]$. Nagelkerke $R^{2}$, a measure of strength of association between the predictors and the dependent variable, was 0.09 for the entire model.

\section{Discussion}

Infertility is a serious time-sensitive disease and associated with physical and psychological distress due to fear of the ultimate inability to have a child. Although short delays in fertility treatment do not appear to affect pregnancy chances [18], any interruption in care delays the pursuit of achieving the goal of conceiving and likely the persistence of, and increase in, psychological distress. Regardless of the reasons for cancellation or delay of treatment, and despite the majority of participants in this study believing they had a greater than $50 \%$ chance of conceiving with fertility treatment, such recommendations for cycle delays would therefore be expected to, and indeed were, be associated with a great deal of psychological distress in our study sample. The initial ASRM
COVID-19 Taskforce recommendations were largely identical to those issued by ESHRE and other societies as well as state mandates that recommended fertility treatments be delayed due to the COVID-19 pandemic. The subsequent delay of fertility treatments in our practice due to the COVID-19 pandemic resulted in psychological distress for all participants in our study and was at a moderate to extreme level for most.

The provision of additional educational materials did not influence participants distress levels related to the recommendations to delay care. While cognitively patients may have agreed that delaying treatment was the safest option, this did not translate to lower levels of distress. Results of regression analyses showed patients who have been trying to conceive for long periods of time without success may be at risk of increased anxiety when cycles are delayed. It is unclear how coping strategies affected distress levels.

It is important to note that more than half of participants agreed with or were neutral about the recommendations to delay care, even while experiencing high levels of distress. As hypothesized, increased education surrounding the rationale for the recommendations improved acceptance of these recommendations for IVF but not for cancellation of ovulation induction cycles, surgeries, and diagnostic procedures. Notably, our educational intervention was focused on IVF and did not include information related to the risks of ovulation induction, surgery, or diagnostic procedures.

Greater acceptance of recommendations to delay care was also observed in patients who were engaged in coping via use of cognitive restructuring, who received the supplemental education, and who had a history of pregnancy loss. This may suggest that patients who engaged in a fact-based analysis were more likely to be cautious about pursuit of family building. Although this fact-based coping strategy was related to increased acceptance of the recommendations, it was not associated with cancellation distress. This shows that while one may accept the facts surrounding treatment related risk, it does not negate the associated distress. 
Supplemental education also did not influence patient's desire to initiate or continue treatment and over half of the patients would have preferred to start or continue care despite the pandemic. The desire to start or continue treatment was positively associated with distress about treatment cancellation/delay, but was not associated with symptoms of anxiety or depression. Although patients may experience symptoms of anxiety and depression, it appears that their specific distress about the current delays to treatment was more salient to decision making. Therefore, it is important for physicians, clinic staff, and mental health professionals to be particularly supportive regarding treatment cancellation distress in addition to attending to patient's overall emotional well-being.

One of the strengths of this study was the randomized inclusion of educational material and the ability to study acceptance and distress comparing two groups. Additionally, the use of validated questionnaires for the measure of anxiety (GAD-7) and depression (PHQ-8) were used in all participants. Another strength was the discrete period of time over which the survey was administered ( 8 days). In a rapidly evolving pandemic, the medical climate was similar for all respondents.

This study is limited by the description of patients from a single fertility center with a demographically homogenous sample which also precluded assessment of group differences by racial/ethnic group However, this study included a large sample size and is currently the only study to assess the effect of supplemental education on acceptance of and distress associated with the pause in fertility treatments due to the COVID19 pandemic. Our response rate is higher than what is seen in most health-related surveys and is considered moderate in nature. Research finds that response rates in this range may not be associated with response bias and that large response rates in smaller samples may pose greater concerns for generalizability [19]. Further, given the similarities in overall acceptance of and psychological distress associated with the recommendations between our study and the previously published work using US patient populations [7, 12] and internationally $[20,21]$ it appears likely that our study results may be generalizable. Finally, although it is possible that participants who were the most distressed could have been more likely to respond to our survey than less distressed participants, our results show that a large percentage of fertility patients experienced distress as a result of the cancellation or delay of fertility care due to COVID-19.

Another limitation of this study was the inability to distinguish whether the depressive and anxious symptoms experienced by the majority of study participants was due to postponing fertility treatment, due to pre-existing distress, or to distress caused by the pandemic itself. These levels of depression of anxiety and depression were higher than what has been previously published using the same measures in infertility patient populations suggesting that the delays to fertility treatment may have affected symptomatology [22, 23]. Further, recent research finds infertility to frequently be more distressing that the COVID-19 pandemic [7] and to negatively affect fertility patient's mental health [24]. Although it is also possible that exposure to the COVID-19 pandemic, in and of itself, resulted in increased psychological symptomatology in our sample $[25,26]$, participants in the current study also reported high levels of distress related to the cancellation/ delay of fertility treatment. We also did not assess whether or not study participants were actively receiving mental health treatment nor whether or not they had been directly affected by the pandemic (e.g., whether or not a loved one had been diagnosed with COVID-19). The collection of such data could have been useful for improving our understanding of participants' emotional experiences.

The current study is unique in that it assessed the role that education placed in acceptance of treatment recommendations and emotional experiences surrounding the recommendations. This study clearly demonstrated that providing detailed rationale behind recommendations improved patient acceptance. This study also showed that despite acceptance, distress was still present. This is not surprising given the literature on giving bad news to patients which highlights the importance of providing concrete information in a compassionate manner as bad news is likely to be distressing [27, 28].

Given the increased risk of emotional distress in patients whose fertility treatments are delayed, the integration of mental health professionals during times of increased distress is warranted. Mental health professionals can be utilized to improve patient coping strategies, provide emotional support, and can partner with physicians to enhance patient education. Finally, we recommend that physician provision of patient education regarding bad news include validation of patients' potential emotional reactions to such news.

Supplementary Information The online version contains supplementary material available at https://doi.org/10.1007/s10815-020-02023-x.

Authors' contributions A.L., D.M., A.S., R.C., E.F., and M.P. designed the survey, D.M. and R.C. collected the data, A.L. and D.M. analyzed the data and wrote the manuscript. A.L., D.M., A.S., R.C., E.F., and M.P. reviewed and edited the final article. All the authors have seen and approved the final version of this manuscript.

Data availability The data that support the findings of this study are available from the corresponding author, MEP, upon reasonable request.

\section{Compliance with ethical standards}

Conflict of interest The authors declare that they have no conflict of interest.

Ethics approval Institutional Review Board (IRB) approval was obtained from Northwestern University. 
Consent to participate The study is an anonymous survey of all patients whose email address was included in the Northwestern Medicine electronic medical record and were seen for treatment or follow-up between February 01, 2020 and April 21, 2020, an email requesting participation in a study constitutes the informed consent.

Code availability Not applicable.

\section{References}

1. Bureau of Labor Statistics. The Employment Situation - April 2020: U.S. Department of Labor; 2020 [Available from: https:/www.bls. gov/news.release/pdf/empsit.pdf. Accessed 8 May 2020.

2. World Health Organization. Coronavirus disease (COVID-19): situation report-105 https://www.who.int/docs/default-source/ coronaviruse/situation-reports/20200504-covid-19-sitrep-105.pdf? sfvrsn=4cdda8af 22020 [Available from: https://www.who.int/ docs/default-source/coronaviruse/situation-reports/20200504covid-19-sitrep-105.pdf?sfvrsn=4cdda8af_2. Accessed 5 May 2020.

3. Wang C, Pan R, Wan X, Tan Y, Xu L, Ho CS, et al. Immediate psychological responses and associated factors during the initial stage of the 2019 coronavirus disease (COVID-19) epidemic among the general population in China. Int J Environ Res Public Health. 2020;17(5).

4. Cao W, Fang Z, Hou G, Han M, Xu X, Dong J, et al. The psychological impact of the COVID-19 epidemic on college students in China. Psychiatry Res. 2020;287:112934.

5. American Society for Reproductive Medicine. Patient Management and Clinical Recommendations During the Coronavirus (COVID19) Pandemic. https://www.asrm.org/globalassets/asrm/asrmcontent/news-and-publications/covid-19/covidtaskforce.pdf2020 [Available from: https://www.asrm.org/globalassets/asrm/asrmcontent/news-and-publications/covid-19/covidtaskforce.pdf. Accessed 5 May 2020.

6. Ananth CV, Baird D, Bloom MS, Bodnar L, Boghossian NS, Chavarro J, et al. A call from reproductive epidemiologists regarding ASRM COVID-19 Task Force Guidelines. Fertility and Sterility Dialogue. 2020 [Available from: https://www. fertstertdialog.com/users/16110-fertility-and-sterility/posts/65728a-call-from-reproductive-epidemiologists-regarding-asrm-covid19-task-force-guidelines\#comment-15534. Accessed 19 June 2020

7. Vaughan DA, Shah JS, Penzias AS, Domar AD, Toth TL. Infertility remains a top stressor despite the COVID-19 pandemic. Reprod BioMed Online. 2020;41:425-7.

8. Farr SL, Anderson JE, Jamieson DJ, Warner L, Macaluso M. Predictors of pregnancy and discontinuation of infertility services among women who received medical help to become pregnant, National Survey of Family Growth, 2002. Fertil Steril. 2009;91(4):988-97.

9. Lawson AK. Psychological stress and fertility. In: Stevenson EL, Hershberger PE, editors. Fertility and Assisted Reproductive Technology (ART): Theory, Research, Policy and Practice For Healthcare Practitioners. 7th ed. New York City: Springer Publishing Company; 2016. p. 65-86.

10. Herbert DL, Lucke JC, Dobson AJ. Depression: an emotional obstacle to seeking medical advice for infertility. Fertil Steril. 2010;94(5):1817-21.
11. Becerra Perez MM, Menear M, Brehaut JC, Légaré F. Extent and predictors of decision regret about health care decisions: a systematic review. Med Decis Mak. 2016;36(6):777-90.

12. Turocy JM, Robles A, Hercz D, D'Alton M, Forman EJ, Williams Z. The emotional impact of the ASRM guidelines on fertility patients during the COVID-19 pandemic. medRxiv. 2020(06/19/ 2020).

13. Ferguson E, Matthews G, Cox T. The appraisal of life events (ALE) scale: Reliability and validity. Br J Health Psychol. 1999;4:97-116.

14. Spitzer RL, Kroenke K, Williams JB, Löwe B. A brief measure for assessing generalized anxiety disorder: the GAD-7. Arch Intern Med. 2006;166(10):1092-7.

15. Kroenke K, Strine TW, Spitzer RL, Williams JB, Berry JT, Mokdad AH. The PHQ-8 as a measure of current depression in the general population. J Affect Disord. 2009;114(1-3):163-73.

16. Kroenke K, Spitzer RL, Williams JBW. The PHQ-9: validity of a brief depression severity measure. J Gen Intern Med. 2001;16(9): 606-13.

17. Morrow KA, Thoreson RW, Penney LL. Predictors of psychological distress among infertility clinic patients. J Consult Clin Psychol. 1995;63(1):163-7.

18. Romanski PA, Bortoletto P, Rosenwaks Z, Schattman GL. Delay in IVF treatment up to 180 days does not affect pregnancy outcomes in women with diminished ovarian reserve. Hum Reprod. 2020;35(7):1630-6.

19. Lie HC, Rueegg CS, Fossa SD, Loge JH, Ruud E, Kiserud CE. Limited evidence of non-response bias despite modest response rate in a nationwide survey of long-term cancer survivors-results from the NOR-CAYACS study. J Cancer Surviv. 2019;13(3):353-63.

20. Boivin J, Harrison C, Mathur R, Burns G, Pericleous-Smith A, Gameiro S. Patient experiences of fertility clinic closure during the COVID-19 pandemic: appraisals, coping and emotions. Hum Reprod. 2020;35:2556-66.

21. Ben-Kimhy R, Youngster M, Medina-Artom TR, Avraham S, Gat I, Haham LM, et al. Fertility patients under COVID-19: attitudes, perceptions, and psychological reactions. Hum Reprod. 2020.

22. Evans-Hoeker EA, Eisenberg E, Diamond MP, Legro RS, Alvero $\mathrm{R}$, Coutifaris $\mathrm{C}$, et al. Major depression, antidepressant use, and male and female fertility. Fertil Steril. 2018;109(5):879-87.

23. Omani-Samani R, Ghaheri A, Navid B, Sepidarkish M, Maroufizadeh S. Prevalence of generalized anxiety disorder and its related factors among infertile patients in Iran: a crosssectional study. Health Qual Life Outcomes. 2018;16(1):129.

24. Gordon JL, Balsom AA. The psychological impact of fertility treatment suspensions during the COVID-19 pandemic. PLoS One. 2020;15(9):e0239253.

25. Choi EPH, Hui BPH, Wan EYF. Depression and anxiety in Hong Kong during COVID-19. Int J Environ Res Public Health. 2020;17(10).

26. Guo J, Feng XL, Wang XH, van IJzendoorn MH. Coping with COVID-19: Exposure to COVID-19 and negative impact on livelihood predict elevated mental health problems in chinese adults. Int J Environ Res Public Health. 2020;17(11).

27. Monden KR, Gentry L, Cox TR. Delivering bad news to patients. Baylor Univ Med Center Proc. 2016;29(1):101-2.

28. Leone D, Menichetti J, Barusi L, Chelo E, Costa M, De Lauretis L, et al. Breaking bad news in assisted reproductive technology: a proposal for guidelines. Reprod Health. 2017;14(1):87.

Publisher's note Springer Nature remains neutral with regard to jurisdictional claims in published maps and institutional affiliations. 